\title{
Diurnal variation of essential and physiological tremor
}

\author{
J J van Hilten, J G van Dijk, R J W Dunnewold, E A van der Velde, B Kemp, \\ $P$ van Brummelen, J A van der Krogt, R A C Roos, O J S Buruma
}

\begin{abstract}
Evaluations were made of the diurnal variations of tremor power at rest, after fatigue and after mass loading, and plasma norepinephrine in patients with familial essential tremor and normal subjects. Diurnal tremor power rhythms for both essential and physiological tremor pursued identical temporal profiles. Plasma norepinephrine levels followed a congruent diurnal pattern with later peak values than the peak values of tremor power. Sympathetic nervous system activity is unlikely to be the cause of diurnal tremor power variation. The consistent diurnal rhythm of tremor power may affect dosage schemes of tremorolytic drugs.
\end{abstract}

Circadian rhythmicity is a common characteristic for almost every physiological variable. Many patients with essential tremor (ET) report diurnal variation of symptoms. ${ }^{23} \mathrm{We}$ studied the diurnal pattern of tremor power at rest, and after fatigue and mass loading in patients with ET and normal subjects with physiological tremor (PT). We also investigated the temporal profile of plasma norepinephrine (PNE) as an indirect measure of sympathetic nervous system activity.

Academic Hospital, Leiden

Department of

Neurology

$\mathrm{J} J$ van Hilten

R A C Roos

O J S Buruma

Department of

Clinical

Neurophysiology

J G van Dijk

R J W Dunnewold

B Kemp

Department of

Nephrology

$P$ van Brummelen

State University,

Leiden, The

Netherlands

Department of

Medical Statistics

E A van der Velde

Department of

Pharmacology

J A van der Krogt

Correspondence to:

Dr van Hilten

Department of Neurology,

Academic Hospital, PO Bo

9600,2300 RC Leiden, The

Netherlands.

Received 12 March 1990

and in revised form

23 July 1990 .

Accepted 7 November 1990

Table Clinical characteristics of essential tremor patients beta-adrenoreceptor antagonists discontinued treatment for at least two weeks before the assessment. All participants abstained from drinks containing caffeine, alcohol or tobacco for at least $\mathbf{1 2}$ hours before the assessment.

\section{Methods}

In all but two participants (one from each group) an iv catheter was inserted into a forearm vein at $07.30 \mathrm{am}$. Collection of blood samples and determination of PNE were performed by methods previously described. ${ }^{4}$ In the course of the day seven recording sessions were carried at 90 minute intervals (from 08.30 to 17.30 ). After 30 minutes relaxation in a supine position, a venous blood sample was drawn followed by a tremor recording. As this study was designed to quantitate diurnal variations of tremor power at baseline conditions, all participants relaxed throughout the study and clinical rating of the tremor amplitude was not performed to avoid the tremor-augmenting effects of mental stress. A light lunch was taken between the third and fourth recording session.

Each recording session consisted of four trials of one minute each. The first two trials were recorded without and the last two trials with $1000 \mathrm{~g}$ mass loading. To evaluate the influence of fatigue all trials were continuous with no rest between the recordings. In ET patients the most tremulous hand was selected for tremor registration and the iv catheter was inserted in the other arm. During the measurements the subjects were seated and rested their pronated forearm on a support, with the wrist on the edge of the support. The hand was thus unsupported. To limit the degree of movement of the hand and fingers to the wrist joint, the fingers were immobilised by using a lightweight clamp (120 g). The clamp consisted of padded dorsal and palmar splints bound together. The clamp has the additional advantage of providing a stable attachment for the accelerometers and for the weights in the

\begin{tabular}{lllll}
\hline Patients & Duration of tremor & Body part involved & Alcohol suppression & $\begin{array}{l}\text { Diurnal fluctuations/crest } \\
\text { time tremor activity }\end{array}$ \\
\hline 1 & 40 & both hands & yes & none \\
2 & 8 & both hands & yes & none \\
3 & 8 & both hands, voice & not known & none \\
4 & 31 & both hands, head & not known & none \\
5 & 20 & both hands & no & none \\
6 & 20 & both hands, head & no & none \\
7 & 21 & both hands & yes & yes/morning \\
8 & 10 & both hands & yes & none \\
9 & 5 & both hands & not known & none \\
10 & 16 & both hands, head & yes & yes/morning \\
\hline
\end{tabular}


loading experiment. Tremor was evaluated with three miniature linear piezo-electric accelerometers (Nihon Kohden, MT-3T), which were attached to the distal end of the clamp, above the fingertips. The accelerometers were placed at right angles to one another, to enable a three-dimensional analysis of movement. The accelerometers had a frequency response extending from a steady state acceleration to $18 \mathrm{~Hz}$ with a sensitivity of a $100 \mathrm{mV}$ output for $1 \mathrm{~g}$ of acceleration. Accelerometer signals were recorded on an Nihon-Kohden EEG signal analyzer. Accelerometer signals were filtered with a high frequency filter of $70 \mathrm{~Hz}$ and a time constant of 1 second. Epochs of 60 seconds were subjected to on-line power spectral analysis. Each analysis was based on 10 Fourier transforms of each 10 seconds. Power for the frequency band from two to $17 \mathrm{~Hz}$ was computed from the power spectra. The combined output of the three accelerometers was used to compute the total power. This total power may be said to represent the amplitude and duration of movements between two and $17 \mathrm{~Hz}$.

\section{Statistical methods}

Because the tremor powers were skewed to the right a logarithmic transformation was applied before further analysis. The resulting variable (log power) is more appropriate for parametric statistical methods, but still represented the amount of tremor. The results were analysed by using regression analysis, analysis of variance (ANOVA) and analysis of covariance (ANCOVA). In the ANOVA a split unit design was used: ${ }^{5}$ the factor subject was considered to be a random factor, which was nested within the fixed factor "groups" and crossed with the fixed factor "session". In this way we simultaneously analysed whether the factors "groups" and "session" and the interaction "groups by session" influenced the results. Accordingly, we investigated differences between both groups for mean tremor power values (taken over all sessions), the existence of diurnal rhythmicity (tremor power and PNE), and differences with respect to their diurnal rhythmicity. $P$ values revealed by this analysis are given in the results together with their meaning as outlined in this section. ANCOVA was used to investigate whether regression lines were parallel or coincident. All tests were performed with a significance level of $p<0.05$.

\section{Results}

\section{Diurnal variance of tremor power}

In ET, the group mean tremor power (GMTP) of each trial in all seven recording sessions was significantly higher than in PT (trial 1-4: $\mathrm{p}=0.002, \mathrm{p}=0.001, \mathrm{p}=0.004, \mathrm{p}=0.003)$. The two groups showed a similar diurnal course of GMTP (ANOVA; significant session effect trial $1 p=0.003$ (fig 1), trial $2 p=0.022$ (fig 1), trial $3 \mathrm{p}=0.004$ (not shown), trial 4 $\mathrm{p}=0.022$ (not shown)). No significant interaction was found between "subject group" and "session number" for any of the four trials.

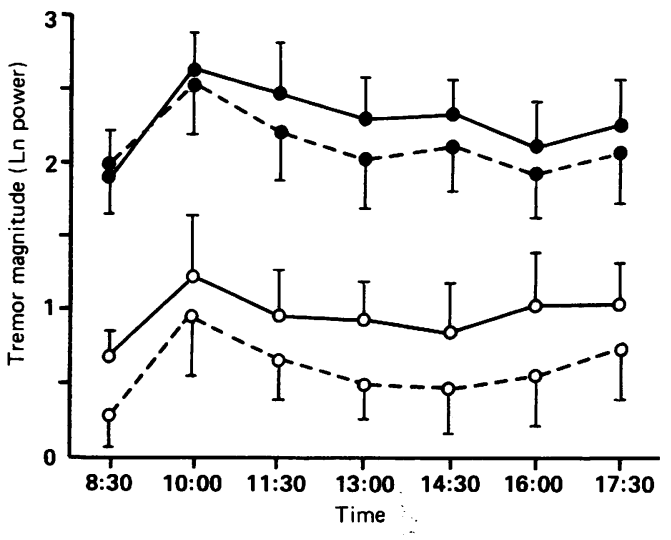

Figure 1 Diurnal profiles of group mean (SE) tremor power of 10 normal subjects $(\bigcirc)$ and 10 patients with ET ( ). The dashed line indicates the first unloaded trial, the full line indicates the second unloaded trial.

\section{Diurnal variance of plasma norepinephrine}

The temporal profile of the group mean PNE (GMPNE) is shown in fig 2. There was no significant difference between the values of the two groups (ANOVA group effect $p=0.856$ ). Both groups clearly demonstrated a similar pattern of diurnal variance (ANOVA; significant session effect $p<0.001$ and no significant interaction between "group" and "session", $p=0.63$ ).

\section{Effect of fatigue and external loading}

The results showed that tremor power followed a similar diurnal course for both groups, in each trial. For further analysis of the effects of fatigue and mass loading, the seven tremor power values of one trial for each subject were added. The sum of powers was thus obtained for all four trials: two unloaded and two loaded trials. The relationships between power values for each subject are shown in figs $3 \mathrm{a}-\mathrm{c}$. In both PT and ET a strong correlation was found between the sum powers of the unloaded trials, and likewise between those of the loaded trials (ANCOVA: $p>0 \cdot 10$ for slope and intercepts for both relationships). After pooling of data of the two groups the regression lines of these relationships are shown in fig $3 a-c$. In addition to the ANCOVA, a Student's $t$ test for paired samples was used to analyse the change in mean tremor power following external loading in the

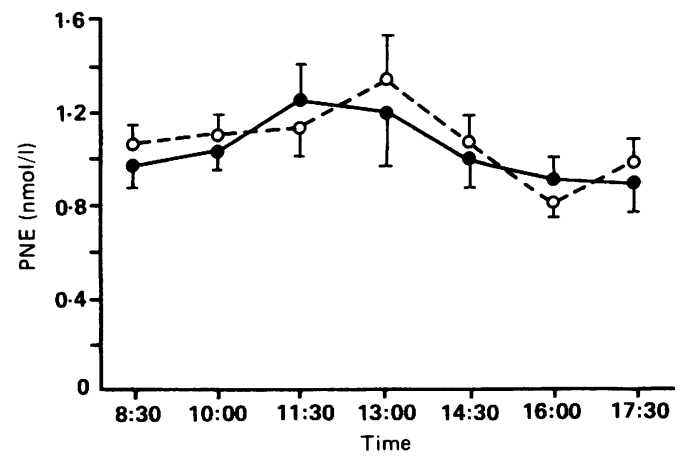

Figure 2 The diurnal profile of the mean level (SE) of plasma norepinephrine (PNE in nmol/L) of nine normal subjects (O) and nine patients with $E T(\Theta)$. 
Figure 3 Graphs illustrating for each normal subject $(\bigcirc)$ and patient with $E T(O)$ the relationship between the sum of unloaded tremor powers of the first trial and the sum of unloaded tremor powers of the second trial $(A)$, sum of unloaded tremor powers of the second trial and the sum of loaded tremor powers of the third trial (B), sum of loaded tremor powers of the third trial and the sum of loaded tremor powers of the fourth trial (C).
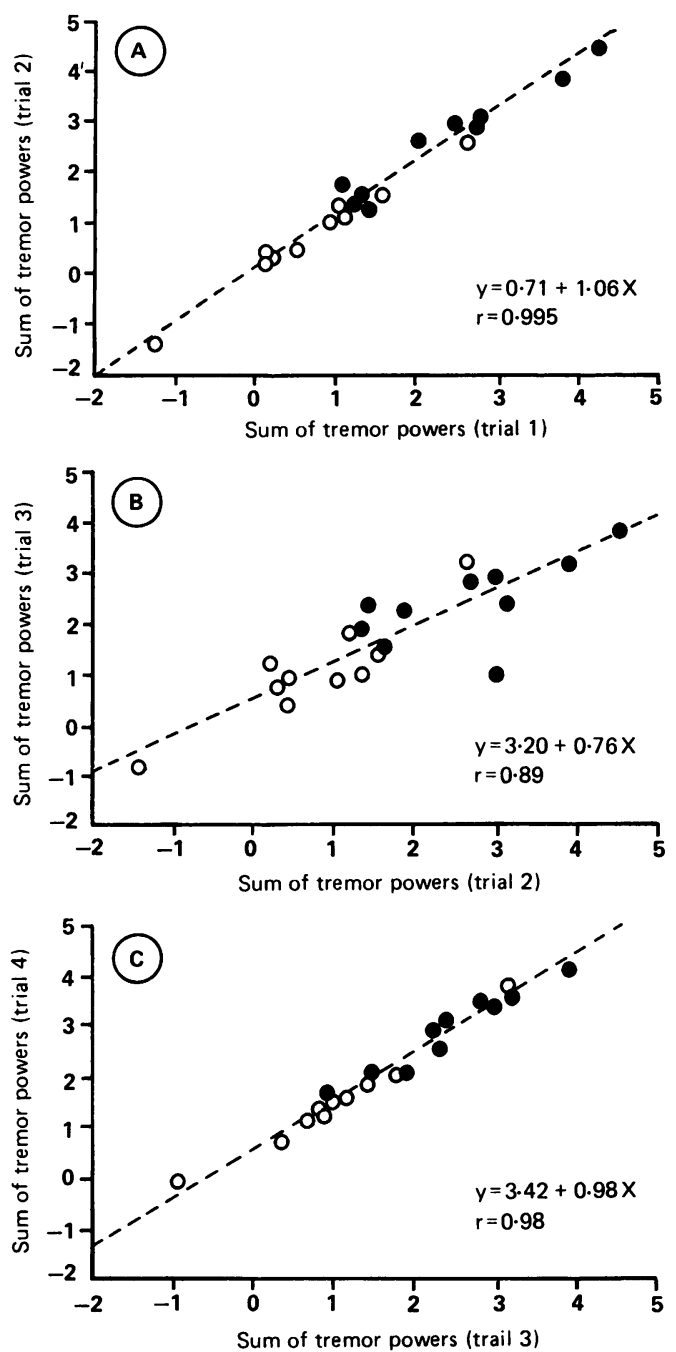

third trial. Without this mass loading, tremor amplitude would probably have increased because of the continued presence of the factor fatigue. However, the PT and ET group showed a mean (SD) change of $1.93(2.79) \mu \mathrm{v}^{2}$, and $-0.78(5.09) \mu \mathrm{v}^{2}$, respectively, which was not significantly different from zero $p=0.06$ and $p=0.64$, respectively. The amount of change was not significantly different for both groups $(p=0 \cdot 16)$. No relationship between tremor power of each session and preceding tremor power or PNE was found (regression analysis) for both groups.

\section{Discussion}

Our results show consistent diurnal rhythms of mean tremor amplitude at rest for both PT and ET with identical temporal profiles. Fatigue (second and fourth trial) followed a similar diurnal course and increase of tremor amplitude for both PT and ET. This effect of fatigue on tremor amplitude has been previously reported in PT. ${ }^{6}$ In the third trial, the effect of mass loading was superimposed on an "enhanced" tremor state. Without this mass loading, tremor amplitude would probably have increased. However, mass loading followed a similar diurnal pattern, and no significant change in amplitude from the previous condition was found. The possibility that a degree of stress accompanying the insertion of the catheter influenced tremor power is unlikely; the lowest tremor power values occurred in the first recording. Furthermore, all concentrations of PNE recorded were within the normal range, rendering a continuous stressful effect of the indwelling catheter during the day unlikely. Considerable controversy still exists concerning the relationship between PT and ET ${ }^{7-10}$ It was, however, not the aim of our study to address this issue and our findings do not allow any conclusion on the role played by central oscillators in the tremor mechanism. They only suggest that the mechanisms, either central or peripheral, responsible for the genesis of PT or ET are influenced by the same circadian pacemaker.

Earlier reports have called attention to the diurnal variation of amplitude in PT. ${ }^{11}$ Others, without specification, reported that PT amplitude was quite variable in the same individual from one time to another. ${ }^{12}$ Our findings accord with those of Tyrer and Bond, who demonstrated a consistent diurnal pattern of amplitude in PT with a maximum at 11.00 am. ${ }^{13}$ Most of the patients with ET who experience diurnal fluctuations in tremor intensity, report it to be worse in the morning. ${ }^{2}$ Koller and Royse observed diurnal variations of amplitude in (untreated) ET as great as $50 \%$. $^{3}$ Half of their patients noted that tremor was worse in the morning. In contrast, Cleeves and Findley observed considerable intra- and inter-individual variation in amplitude of untreated ET, but no difference in mean tremor levels in a diurnal study. ${ }^{14}$ In their study, however, patients were examined in six-hourly assessments and the hour of each assessment was not specified. Furthermore, as many extraneous factors are known to influence tremor amplitude, these might also interfere with the expression of the diurnal rhythm. In view of this, common daily activities are likely to result in irregular diurnal fluctuations of tremor amplitude, and intrinsic diurnal rhythmicity can become overt only when properly measured at frequent intervals under controlled experimental conditions.

The circadian rhythm of plasma catecholamine levels, thought to reflect variations in sympathetic nervous system activity, shows a gradual rise during the morning hours and a peak around noon. ${ }^{15}$ Circadian plasma catecholamine rhythms are thought to be related to diurnal variations of blood pressure and heart rate. ${ }^{1617}$ Furthermore, recent epidemiological studies on ET revealed an intriguing association with hypertension. ${ }^{18}$ Much of the data on cathecholamine effects relate to actions of epinephrine, not norepinephrine. ${ }^{19}{ }^{20}$ Additionally, these studies showed that only plasma levels of epinephrine comparable with those seen during stress and exercise caused an increase in tremor amplitude. Our main interest was whether tremor amplitude at rest was related to PNE as an indirect measure of sympathetic nervous system activity. ${ }^{21}$ It is unlikely that lunch taken between 11.30 am and 13.00 had any effect on the GMPNE; the peak value of the GMPNE of the ET group occurred 
before lunch and a significant increase of PNE after meals could not be shown in one previous study. ${ }^{22}$ We found a similar mean diurnal PNE value and phase-relationship with tremor power for ET and PT. The diurnal peaks of GMTP and GMPNE show apparent phasedifferences which are of some functional interest. Since the diurnal rhythm of GMTP was phase-advanced with respect to GMPNE, the temporal profile of tremor power cannot be explained by the diurnal variations of PNE. This finding may indicate that baseline tremor power is not related to sympathetic nervous system activity.

In our study evidence was presented showing the diurnal nature of tremor amplitude. Knowledge of a rhythmic pattern of symptoms can be useful in selecting the most beneficial therapeutic regimen. Against this background, the use of chronobiological methods to investigate dosing schedules should be explored.

We are grateful to A R Wintzen for his critical comments, Vera Reijns for technical assistance and Janita Molman for secretarial help.

1 Moore-Ede MC, Czeisler CA, Richardson GS. Circadian timekeeping in health and disease. Part 1. Basic properties 2 Gerstendbrand F, Klinger D, Pfeiffer B. Der essentiale Tremor, phanomenologie und epidemiologie. Nervenarz 982;43:46-53.

3 Koller WC, Royse VL. Time course of a single oral dose of propanolol in essential tremor. Neurology 1985;35:1494-8.

4 Chang PC, van der Krogt JA, van Brummelen $P$ Demonstration of neuronal and extraneuronal uptake of circulating norepinephrine in the forearm. Hypertension 1987;9:647-53.
5 Armitage P, Berry G. Statistical methods in medical research. 2nd ed. Oxford: Blackwell Scientific, 1987.

6 Stiles RN. Mechanical and neural feedback factors in postural hand tremor of normal subjects. J Neurophysiol 1980;44:40-59.

7 Marshall J. Observations on essential tremor. J Neurol Neurosurg Psychiatry 1962;25:122-25.

8 Marshall J. The effects of ageing upon physiological tremor. $J$ Neurol Neurosurg Psychiatry 1961;24:14-17.

9 Elble RJ. Physiologic and essential tremor. Neurology 1986;36:225-31.

10 Marsden CD, Obeso A, Rothwell JC. Benign essential tremor is not a single entity. In: Yahr MD, ed. Current concepts of Parkinson's disease and related disorders. Amsterdam: Exerpta Medica, 1983:31-46.

11 Marsden CD, Meadows JC, Lange GW, Watson RS Variations in human physiological finger tremor, with particular reference to changes with age. Electroenceph Clin Neurophysiol 1969;27:169-78.

12 Shahani BT, Young RR. Physiological and pharmalogical aids in the differential diagnosis of tremor. J Neurol aids in the differential diagnosis of

13 Tyrer PJ, Bond AJ. Diurnal variation in physiological tremor. Electroenceph Clin Neurophysiol 1974;37:35-40.

14 Cleeves L, Findley LJ. Variability in amplitude of untreated essential tremor. J Neurol Neurosurg Psychiatry 1987;50:704-8.

15 Turton MB, Deegan T. Circadian variations of plasma catecholamine, cortisol and immunoreactive insulin concentrations in supine subjects. Clin Chim Acta 1974;55:389-97.

16 Millar-Craig MW, Bishop CN, Raftery. EB. Circadian variation of blood-pressure. Lancet 1978;i:795-7.

17 Sirgo MA, Mills RJ. DeQuattro V. Effects of antihypertensive agents on circadian blood pressure and heart rate patterns. Arch Intern Med 1988;148:2547-52.

18 Larsen T, Calne DB. Essential tremor. Clin Neuropharmacol 1983;6:185-206.

19 Esler M, Willett J, Leonard P, et al. Plasma noradrenaline kinetics in humans. J Auton Nerv Syst 1984;11:125-44.

20 Marsden CD, Foley TH, Owen AL. Peripheral B-adrenergic receptors concerned with tremor. Clin Sci 1967;33 53-65.

21 Fellows IW, Macdonald IA, Wharrad HJ. Low plasma concentrations of adrenaline and physiological tremor in man. J Neurol Neurosurg Psychiatry 1986;49:396-9.

22 Mullen PE, Lightman S, Linsell P, McKeon P, Sever PS Todd $\mathrm{K}$. Rhythms of plasma noradrenaline in man. Psychoneuroendocrinology 1981;6:213-22. 